\title{
Widespread inflammation in CLIPPERS syndrome indicated by autopsy and ultra-high-field 7T MRI \\ OPEN
}

Morten Blaabjerg, MD, $\mathrm{PhD}$ *

Klemens Ruprecht, MD* Tim Sinnecker, MD

Daniel Kondziella, MD, $\mathrm{PhD}$

Thoralf Niendorf, PhD Bjørg Morell Kerrn-

Jespersen, MD

Mette Lindelof, MD

Hans Lassmann, MD

Bjarne Winther

Kristensen, MD, PhD

Friedemann Paul, MD $\ddagger$

Zsolt Illes, MD, PhD

Correspondence to

Dr. Illes:

zsolt.illes@rsyd.dk

Supplemental data at Neurology.org/nn

\section{ABSTRACT}

Objective: To examine if there is widespread inflammation in the brain of patients with chronic lymphocytic inflammation with pontine perivascular enhancement responsive to steroids (CLIPPERS) syndrome by using histology and ultra-high-field MRI at 7.OT.

Methods: We performed a detailed neuropathologic examination in 4 cases, including 1 autopsy case, and studied 2 additional patients by MRI at 7.0T to examine (1) extension of inflammation to areas appearing normal on 3.0T MRI, (2) potential advantages of 7.OT MRI compared to 3.OT MRI in reflecting widespread inflammation, perivascular pathology, and axonal damage, and (3) the possibility of lymphoma.

Results: In the autopsy case, perivascular inflammation dominated by CD4 + T cells was not only detected in the brainstem and cerebellum but also in brain areas with normal appearance on 3.OT $\mathrm{MRI}$, including supratentorial regions and cranial nerve roots. There was no evidence of lymphoma in any of the 4 patients. The 7.OT MRI in clinical remission also revealed supratentorial lesions and perivascular pathology in vivo with contrast-enhancing lesions centered around a small venous vessel. Ultra-high-field MRI at 7.0T disclosed prominent T1 hypointensities in the brainstem, which were not seen on 3.0T MRI. This corresponded to neuropathologic detection of axonal injury in the autopsy case.

Conclusion: Our findings suggest more widespread perivascular inflammation and postinflammatory axonal injury in patients with CLIPPERS. Neurol Neuroimmunol Neuroinflamm 2016;3:e226; doi: 10.1212/NXI.0000000000000226

\section{GLOSSARY}

CLIPPERS = chronic lymphocytic inflammation with pontine perivascular enhancement responsive to steroids; $\mathbf{H} \& \mathbf{E}=$ hematoxylin \& eosin; $\mathbf{m R S}=$ modified Rankin Scale; $\mathbf{M S}=$ multiple sclerosis; $\mathbf{T} 2 * \mathbf{W}=$ T2*-weighted; TE $=$ echo time; TR = repetition time; T1W MPRAGE $=$ T1-weighted magnetization-prepared rapid gradient echo; $\mathbf{S W I}=$ susceptibilityweighted imaging; VIBE = volumetric interpolated brain examination.

Features of chronic lymphocytic inflammation with pontine perivascular enhancement responsive to steroids (CLIPPERS) ${ }^{1}$ include subacute brainstem symptoms, MRI appearance with multiple punctate or curvilinear gadolinium enhancements, and a clear response to immunosuppressive treatment. ${ }^{1-3}$ Since the original case series, several cases have been described, some of which suggest that the CLIPPERS MRI appearance can be seen in other inflammatory disorders, such as primary angiitis of the $\mathrm{CNS}^{4}$ or multiple sclerosis (MS). ${ }^{5}$ Recently, cases have been reported with development of lymphoma after the initial diagnosis of CLIPPERS. ${ }^{6-8}$

\footnotetext{
*These authors contributed equally to this work.

$\ddagger$ These senior authors contributed equally to this work.

From the Departments of Neurology (M.B., Z.I.) and Pathology (B.W.K.), Odense University Hospital, Denmark; Clinical and Experimental Multiple Sclerosis Research Center (K.R., T.S., F.P.), Department of Neurology (K.R., F.P.), NeuroCure Clinical Research Center (T.S., F.P.), and Experimental and Clinical Research Center (T.N., F.P.), Charité-Universitätsmedizin Berlin, Germany; Department of Neurology (T.S.), Universitatsspital Basel, Switzerland; Department of Neurology (D.K.), Rigshospitalet, Copenhagen University Hospital, Denmark; Berlin Ultrahigh Field Facility (B.U.F.F.) (T.N.), Max Delbrueck Center for Molecular Medicine (F.P.), Berlin, Germany; Department of Neurology (B.M.K.-J., M.L.), Herlev Hospital, Denmark; Center for Brain Research (H.L.), Medical University of Vienna, Austria; and Institute of Clinical Research (M.B., B.W.K., Z.I.), University of Southern Denmark, Odense.

Funding information and disclosures are provided at the end of the article. Go to Neurology.org/nn for full disclosure forms. The Article Processing Charge was paid by Odence University Hospital.

This is an open access article distributed under the terms of the Creative Commons Attribution-NonCommercial-NoDerivatives License 4.0 (CC BY-NC-ND), which permits downloading and sharing the work provided it is properly cited. The work cannot be changed in any way or used commercially.
} 
While former neuropathologic examinations have revealed a predominant perivascular $\mathrm{CD} 3+\mathrm{T}$-cell inflammation in the affected regions, these studies were confined to biopsy specimens and thus could not analyze the extent of inflammation in other brain areas. Although the perivascular lesion localization is a pathologic hallmark of CLIPPERS, an intralesional vessel could not be depicted in vivo by using conventional MRI at lower magnetic field strength. Gaining from increased susceptibility effects and spatial resolution, ultra-high-field MRI at 7.0T improved the detection of both vascular abnormalities ${ }^{9-13}$ and structural CNS damage ${ }^{14-16}$ and may thus be a promising technique to visualize perivascular inflammation and tissue destruction in CLIPPERS.

We report results of a neuropathologic examination of an autopsy case to study the pathology of brain regions with normal appearance on 3.0T MRI. We also investigated supratentorial lesions in 2 patients with CLIPPERS in vivo by 7.0T MRI.

\section{METHODS Standard approvals, registrations, and patients. Biopsy samples were obtained for diagnostic purposes. Pathologic samples (biopsy and autopsy) were retrospectively analyzed. The pro- spective MRI part of the study was approved by the local ethics com- mittee (EA 1/054/09). Written consent was obtained from all participants prior to examination.}

Description of autopsy case (patient 1). Patient 1 is a 62year old man who was admitted in 2008 because of subacute onset diplopia, dysphagia, and balance problems (table). Neurologic examination revealed gaze-evoked nystagmus, diplopia, limb and gait ataxia, and moderate postural tremor of the upper extremities. MRI showed patchy T2 signal hyperintensities in the brainstem and cerebellar peduncles with punctate gadolinium enhancements (figure 1). Three lumbar punctures during the disease course revealed mild lymphocytic pleocytosis $(37,17$, and 15 leukocytes per microliter) but no oligoclonal bands. MRI angiography and whole-body FDGPET-CT were normal. There was no evidence of malignant cells and viral examinations of the CSF for herpesviruses were negative. Search for onconeural antibodies, antinuclear antibodies, antineutrophil cytoplasmic antibodies, anti-SSA, antiSSB, anticardiolipin, elevated angiotensin-converting enzyme in sera and CSF, and tuberculosis tests were normal. The patient recovered on high-dose steroid treatment, but relapsed after its withdrawal. Brainstem biopsy showed perivascular CD4 + T-cell infiltrations. There was no evidence of lymphoma or vessel destruction. Staining for herpes simplex virus 1 and 2 were normal. The patient responded to steroid treatment, but then had a fatal brainstem hemorrhage. In 2014, the history and initial brain biopsy specimens were re-evaluated and CLIPPERS syndrome was diagnosed.

Description of the 3 cases with brainstem biopsy (patients 2-4). The clinical course, MRI data, and follow-up of the 3 patients have been recently described elsewhere. ${ }^{17}$ Patient 2 is a 23-year-old man who developed subacute nystagmus and gait ataxia (table). He was treated with methylprednisolone and azathioprine, but had 6 relapses in 14 years with gradual worsening of cerebellar dysarthria and gait ataxia. CSF showed mild pleocytosis and oligoclonal bands. Brain biopsy indicated perivascular inflammatory infiltrates predominantly composed of CD3 $+\mathrm{T}$ cells. MRI showed punctate gadolinium enhancement in the pons. No systemic autoantibodies were found. At present, the patient is ambulatory (modified Rankin Scale [mRS] 2). ${ }^{17}$ Patient 3 is a 58-year-old woman who developed subacute gait ataxia, dysarthria, and paresthesia of the face, hands, and feet (table). She had 4 relapses in 6 years. CSF showed mild lymphocytosis and no oligoclonal bands. Brain biopsy indicated parenchymal and perivascular inflammatory infiltrates predominantly composed of $\mathrm{CD} 3+\mathrm{T}$ cells. MRI revealed multiple punctate gadolinium enhancement in the pons and brachium pontis. No systemic autoantibodies were found. At present, the patient is severely dysarthric, wheelchair-bound, and has mild involuntary movements (mRS 4). ${ }^{17}$ Patient 4 is a 42 -year-old man who

\begin{tabular}{|c|c|c|c|c|c|c|c|c|}
\hline \multirow[b]{2}{*}{ Patients } & \multirow[b]{2}{*}{ Sex/age, y } & \multirow[b]{2}{*}{ Time and symptoms at diagnosis } & \multicolumn{2}{|c|}{$\begin{array}{l}\text { Clinical } \\
\text { state at } \\
\text { MRI }\end{array}$} & \multirow[b]{2}{*}{$\begin{array}{l}\text { Treatment at } \\
\text { biopsy }\end{array}$} & \multirow[b]{2}{*}{$\begin{array}{l}\text { Treatment at } \\
\text { MRI }\end{array}$} & \multirow[b]{2}{*}{$\begin{array}{l}\text { Time between symptom onset } \\
\text { and MRI/autopsy/biopsy }\end{array}$} & \multirow[b]{2}{*}{ Ref } \\
\hline & & & 3.0T & 7.0T & & & & \\
\hline $\begin{array}{l}\text { Patient } 1 \text { (autopsy } \\
\text { and 3.0T MRI) }\end{array}$ & $M / 62$ & $\begin{array}{l}\text { 2008: Diplopia, dysphagia, dysarthria, } \\
\text { ataxia }\end{array}$ & Rel & NA & Prednisone & Prednisone & $\begin{array}{l}\text { 3.0T MRI: } 1 \text { week; autopsy: } \\
3 \text { mo }\end{array}$ & NA \\
\hline Patient 2 (biopsy 1) & $M / 23$ & 1999: Nystagmus, ataxia, anorexia & NA & NA & None & NA & Biopsy: 27 mo & 17 \\
\hline Patient 4 (biopsy 3) & $\mathrm{M} / 42$ & $\begin{array}{l}\text { 2010: Tetraspasticity, proximal } \\
\text { paraparesis, dysarthria, diplopia, ataxia }\end{array}$ & NA & NA & Prednisone & NA & Biopsy: 5 mo & 17 \\
\hline $\begin{array}{l}\text { Patient } 5 \text { (3.0T and } \\
\text { 7.0T MRI) }\end{array}$ & $F / 48$ & $\begin{array}{l}\text { 2013: Hemiparesthesia, double vision, } \\
\text { gait instability, dysarthria, facial pain }\end{array}$ & Rem & Rem & NA & $\begin{array}{l}\text { Prednisone; } \\
\text { methotrexate }\end{array}$ & 7.0T MRI: 13 mo & NA \\
\hline $\begin{array}{l}\text { Patient } 6 \text { (3.0T and } \\
\text { 7.0T MRI) }\end{array}$ & F/73 & 2010: Fatigue, diplopia, ataxia, dysarthria & Rem & Rem & NA & $\begin{array}{l}\text { Prednisone; } \\
\text { methotrexate }\end{array}$ & $\begin{array}{l}\text { 3.OT MRI: } 49 \text { mo; } 7.0 T \text { MRI: } \\
49 \text { mo }\end{array}$ & 18 \\
\hline
\end{tabular}

Abbreviations: NA = not applicable; Rel = Relapse; Rem = Remission. 

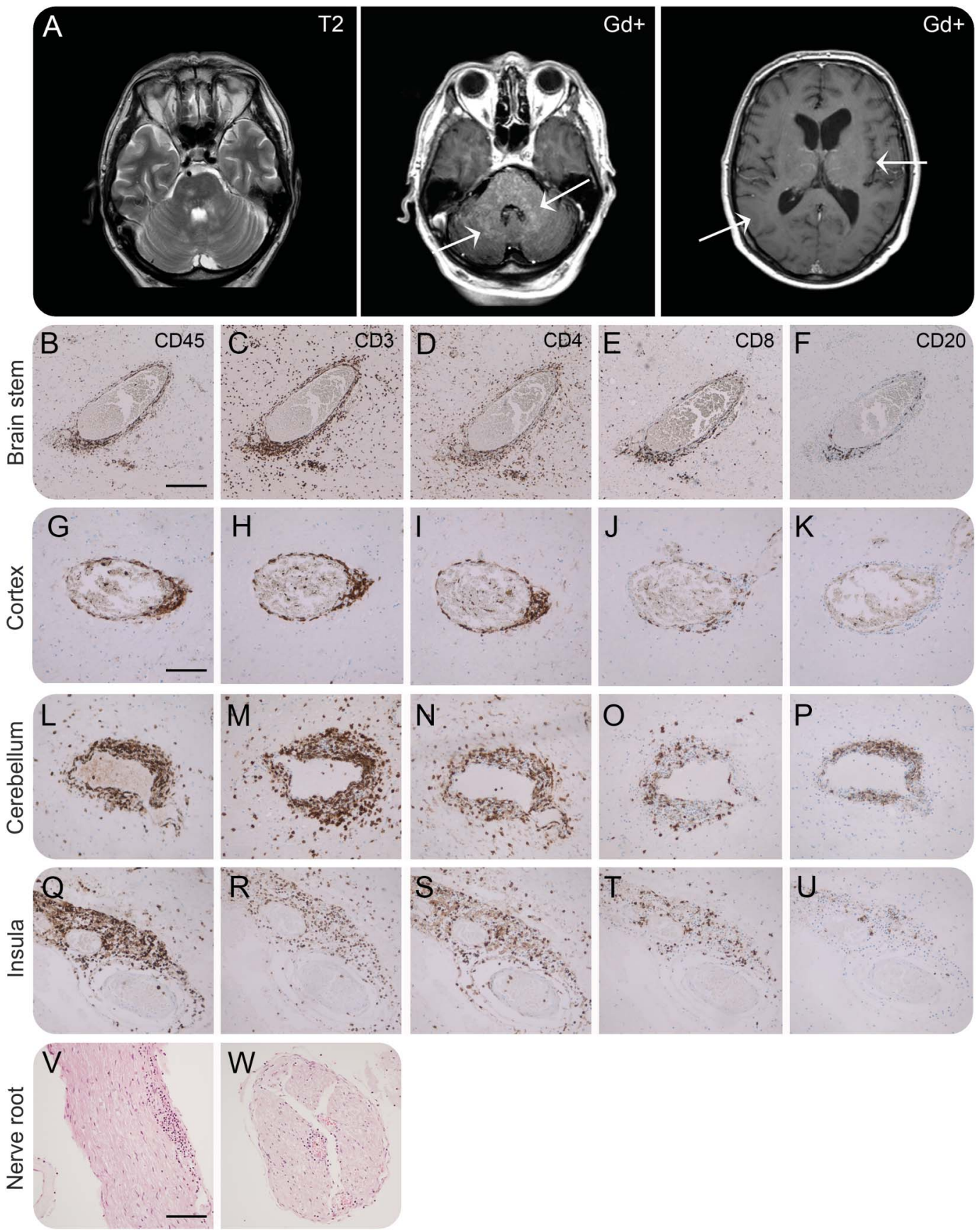

(A) MRI of patient 1 shows T2-weighted hyperintensities in brainstem and cerebellar peduncles with similar pattern of gadolinium enhancement. Arrows depict areas used for immunohistochemical stainings for CD45, CD3, CD4, CD8, and CD20 in brainstem (B-F) and cerebellum (L-P) with punctate gadolinium enhancements and parietal (G-K) and insular cortex (Q-U) with normal MRI appearance. We found prominent CD4+ T-cell infiltration in the brainstem (D) and cerebellum (N) but also in insular cortex (S) and parietal cortex (I). The magnitude of infiltration shows a gradient with less infiltration with greater distance from the brainstem. Similarly, we found less infiltration of CD8 $+T$ cells $(E, J, O$, and $T$ ) and only a very limited number of $\mathrm{CD} 20+\mathrm{B}$ cells $(\mathrm{F}, \mathrm{K}, \mathrm{P}$, and $\mathrm{U})$ with a similar gradient in cell numbers and degree of infiltration into the tissue. Hematoxylin \& eosin staining reveals inflammation also in cranial nerve roots from the brainstem ( $V$ and $W$ ). Magnification $5 \times(B$ and F) (V and W), 10× (G-U). Scale bar: $200 \mu \mathrm{m}(B-F), 100 \mu \mathrm{m}(\mathrm{G}-\mathrm{U}), 200 \mu \mathrm{m}(\mathrm{V}, \mathrm{W})$.

developed subacute dysarthria, horizontal nystagmus, diplopia, jerky eye movements, ataxia, tetraspasticity, and paraparesis (table). CSF showed mild pleocytosis and no persisting oligoclonal bands. Brain biopsy indicated parenchymal and perivascular inflammatory infiltrates predominantly composed of
CD3 $+\mathrm{T}$ cells. MRI showed multiple punctate gadoliniumenhancing lesions in the cerebellum, brainstem, and midbrain. No systemic autoantibodies were found. Symptoms and MRI lesions rapidly responded to methylprednisolone. He was treated with methotrexate and azathioprine, and had only a single relapse 
during the follow-up period of 19 months (mRS 2). The patient committed suicide. ${ }^{17}$

Patients examined by 7T MRI (patients 5 and 6). Two additional patients with CLIPPERS were included in the 7.0T MRI study.

Patient 5 is a 48 -year-old woman who developed subacute numbness in the left half of her body, double vision, gait instability, dysarthria, facial pain, numbness of the oral cavity, and fatigue over a period of 2 weeks in September 2013 (table). During the weeks before the onset of these symptoms, she had noted frontal headaches. The patient was admitted to another hospital in October 2013, where a cranial 1.5T MRI demonstrated multiple small T2 hyperintense lesions with punctate and curvilinear contrast enhancement in the pons and cerebellum, and a bigger right cerebellar lesion. As this case has not been published before, the brain MRI findings of this patient are shown in figure e-1 at Neurology.org/nn. A spinal cord MRI demonstrated a small longitudinal contrast-enhancing lesion in the cervical cord. The cerebellar lesion was biopsied in October 2013. Histologic analysis revealed cerebellitis with prominent CD4 + T-cellular infiltrates, compatible with CLIPPERS syndrome. There were no signs of lymphoma. Antinuclear antibodies, antibodies to extractable nuclear antigens, and antineutrophil cytoplasmic antibodies as well as antibodies to aquaporin- 4 were negative. She was initially treated with high-dose IV methylprednisolone $(1 \mathrm{~g} /$ day for 3 days followed by $1 \mathrm{~g} /$ day for 5 days) and subsequently with oral prednisolone (starting dose $25 \mathrm{mg}$ /day with a subsequent taper to $5 \mathrm{mg} /$ day $)$ and azathioprine $(3 \times 50 \mathrm{mg})$, which was changed to methotrexate in May 2014, when a cranial MRI demonstrated constant to minimally progressive CLIPPERS lesions. At the time of the 7.0T MRI examination, she was treated with prednisolone (5 mg daily) and methotrexate (15 mg weekly), and had residual facial paresthesiae but no clinical signs of new disease activity.

Patient 6 is a 73-year-old woman who was diagnosed with CLIPPERS in October 2010 and whose clinical and paraclinical details were previously reported in detail. ${ }^{18}$ The patient had mildly elevated antinuclear antibodies (1:320) and doublestranded DNA antibodies (immunoglobulin G). Antibodies against aquaporin- 4 and extractable nuclear antigens were negative. At the time of the 7.0T MRI examination, she was treated with methylprednisolone ( $4 \mathrm{mg}$ daily) and methotrexate $(15 \mathrm{mg}$ weekly) and was in clinical remission.

Neuropathologic examination. Three-micrometer sections from formalin-fixed, paraffin-embedded tissue blocks were stained with hematoxylin \& eosin (H\&E) and for CD45 (clone: 2B11\&PD7/26 ventana-LCA, Ventana Medical Systems, Tucson, AZ), CD3 (clone: 2GV6, Ventana Medical Systems), CD4 (SP35, Ventana Medical Systems), CD8 (clone: C8/144B, Dako, Glostrup, Denmark), CD20 (clone: cyL26, Ventana Medical Systems), and neurofilament heavy-chain (NF smi31, Affinity, Exeter, UK). Selected sections from brainstem and insular cortex were double-stained for $\mathrm{CD} 3$ and NF. Adjacent sections were stained for Luxol fast blue and H\&E. Adjacent formalin-fixed tissue section from brainstem tissue (autopsy) as well as sections from 3 additional brainstem biopsies were similarly stained for light-chain restriction (kappasmi and lambda-smi, smlg reaction, Dako), the zinc-finger transcription factor, B-cell lymphoma 6 protein (Bcl-6, clone: LN22 NovoCastra, Leica Biosystems, Newcastle, UK), the large B-cell lymphoma marker, Multiple Myeloma Oncogene 1 (MUM-1, IRF4, Dako), and the precursor B lymphoma marker, CD10 (clone: 56C6-CALLA, NovoCastra, Leica
Biosystems). All immunohistochemical stainings were performed using the BenchMark Ultra IHC staining system (Ventana Medical Systems). For detection, ultraView Universal DAB Detection Kit was applied (Ventana Medical Systems). Toluidine blue was used as counterstaining. Lymphoma stainings were assessed by a skilled lymphoma pathologist. Digital images were obtained at a magnification of $5 \times$ or $10 \times$ (brainstem) or $10 \times$ (cerebellum, parietal cortex, insular cortex/basal ganglia, nerve root, and biopsies 1-3) using a Leica microscope (Leica 4000B LED, Leica Microsystems, Wetzlar, Germany) equipped with a Leica digital camera (Leica DFC420, Leica Microsystems).

Ultra-high-field MRI data acquisition and postprocessing. Ultra-high-field MRI were acquired using a 7.0T whole body MRI scanner (Magnetom, Siemens, Erlangen, Germany) applying a 24-channel receive head coil (Nova Medical, Wilmington, MA) equipped with a birdcage volume coil used for transmission. The imaging protocol included 2D $\mathrm{T} 2{ }^{*}$ weighted $(\mathrm{T} 2 * \mathrm{~W})$ fast low angle shot (echo time $[\mathrm{TE}]=$ $25.0 \mathrm{~ms}$, repetition time $[\mathrm{TR}]=1,820 \mathrm{~ms}$, spatial resolution $=$ $0.2 \times 0.2 \times 2 \mathrm{~mm}^{3}$, supratentorial coverage), pregadolinium and postgadolinium 3D T1-weighted magnetization-prepared rapid gradient echo (T1W MPRAGE) (TE $=2.98 \mathrm{~ms}$, $\mathrm{TR}=2,300 \mathrm{~ms}$, inversion time $=900 \mathrm{~ms}$, spatial resolution $=1.0 \times 1.0 \times 1.0 \mathrm{~mm}^{3}$, whole brain coverage), and $3 \mathrm{D}$ flow-compensated gradient echo-based susceptibilityweighted imaging (SWI) $(\mathrm{TE}=14 \mathrm{~ms}, \mathrm{TR}=25 \mathrm{~ms}$, flip angle $=12^{\circ}$, spatial resolution $=0.5 \times 0.5 \times 1.0 \mathrm{~mm}^{3}$, supratentorial and infratentorial coverage). For infratentorial imaging, postgadolinium volumetric interpolated brain examination (VIBE) sequence was used ( $\mathrm{TE}=3.3 \mathrm{~ms}$, $\mathrm{TR}=19.7 \mathrm{~ms}$, spatial resolution $\left.=0.5 \times 0.5 \times 1.0 \mathrm{~mm}^{3}\right)$.

To identify vascular structures within contrast-enhancing CLIPPERS lesions, we calculated the sum of (1) coregistered T1W MPRAGE with supratentorial T2*W images, and (2) coregistered VIBE with infratentorial SWI images using the image calculator function of the MIPAV software package (version 7.0.1, Bethesda, MD).

RESULTS Perivascular inflammation in CLIPPERS extends to areas with normal appearance on 3.0T MRI: Analysis of the autopsy case. First, we investigated inflammation in different brain regions corresponding to gadolinium-enhancing and normal-appearing brain areas on 3.0T MRI (autopsy tissue of patient 1): brainstem and cerebellum were compared to parietal and insular cortex (figure 1). Perivascular accumulation of CD45+ lymphocytes (figure 1, B, G, L, and Q) with predominance of CD3+ $\mathrm{T}$ cells (figure 1, C, H, M and R) and CD4+ $\mathrm{T}$ cells were detected in brainstem and cerebellum as expected (figure 1, D and N). CD4+ T cells also infiltrated the parenchyma, which was most pronounced in the brainstem (figure 1D). Accumulation and infiltration by CD8 $+\mathrm{T}$ cells were less pronounced (figure 1, E and Q), and only a few CD20 + B cells were observed in close proximity to the vessel wall (figure $1, \mathrm{~F}$ and $\mathrm{P}$ ). However, insular and parietal cortex with normal appearance on 3.0T MRI revealed a similar, albeit less prominent perivascular accumulation of CD4+ 
$\mathrm{T}$ cells (figure 1, I and S), fewer CD8 $+\mathrm{T}$ cells (figure $1, \mathrm{~J}$ and $\mathrm{T}$ ), and a very limited number of $\mathrm{CD} 20+\mathrm{B}$ cells (figure $1, \mathrm{~K}$ and $\mathrm{U}) . \mathrm{CD} 4+\mathrm{T}$ cells also infiltrated the parenchyma the insular cortex (figure 1S). Perivascular inflammation was also found in the roots of cranial nerves (figure 1, $\mathrm{V}$ and $\mathrm{W}$ ). These data suggested that brain areas with normal appearance on 3.0T MRI may be affected by inflammation in CLIPPERS syndrome.

No evidence of lymphoma and prelymphoma state: Analysis of $\mathbf{4}$ cases. Next, brainstem samples (patients 1-4) were analyzed for the possibility of lymphoma or prelymphoma. We found no evidence for monoclonal cell expansion using staining for light chain restriction (figure 2, A-B, F-G, K-L, and P-Q) and lymphoma markers CD10, Bcl-6, and MUM-1 (figure 2, C-E, H-J, M-O, and R-T). Follow-up of the 3 biopsy cases (patients 2-4; mean of 75 months) did not indicate clinical or paraclinical evidence of lymphoma either.

Ultra-high-field MRI reveals perivascular lesions outside the brainstem/cerebellum and tissue damage. Since pathologic examination indicated perivascular inflammation in brain areas with normal appearance on 3.0T MRI, albeit less pronounced than in brainstem and cerebellum, we next examined 2 patients with CLIPPERS by 7.0T MRI during clinical remission. Contrast-enhancing lesions $(\mathrm{n}=20)$ were only depicted in patient 5. Of those, 2 lesions were found in a supratentorial localization affecting ( $\mathrm{n}=$ 1 ) or bordering $(n=1)$ the thalamus (figure $3 \mathrm{D})$. Cortical gray matter lesions were not observed on supratentorial $\mathrm{T} 2 * \mathrm{~W}$ images.

To visualize the perivascular distribution of lesions, we fused contrast-enhanced T1W and supratentorial $\mathrm{T} 2 * \mathrm{~W}$ images with a volume resolution

Figure 2 Neuropathologic examination for lymphoma etiology (patients 1-4)
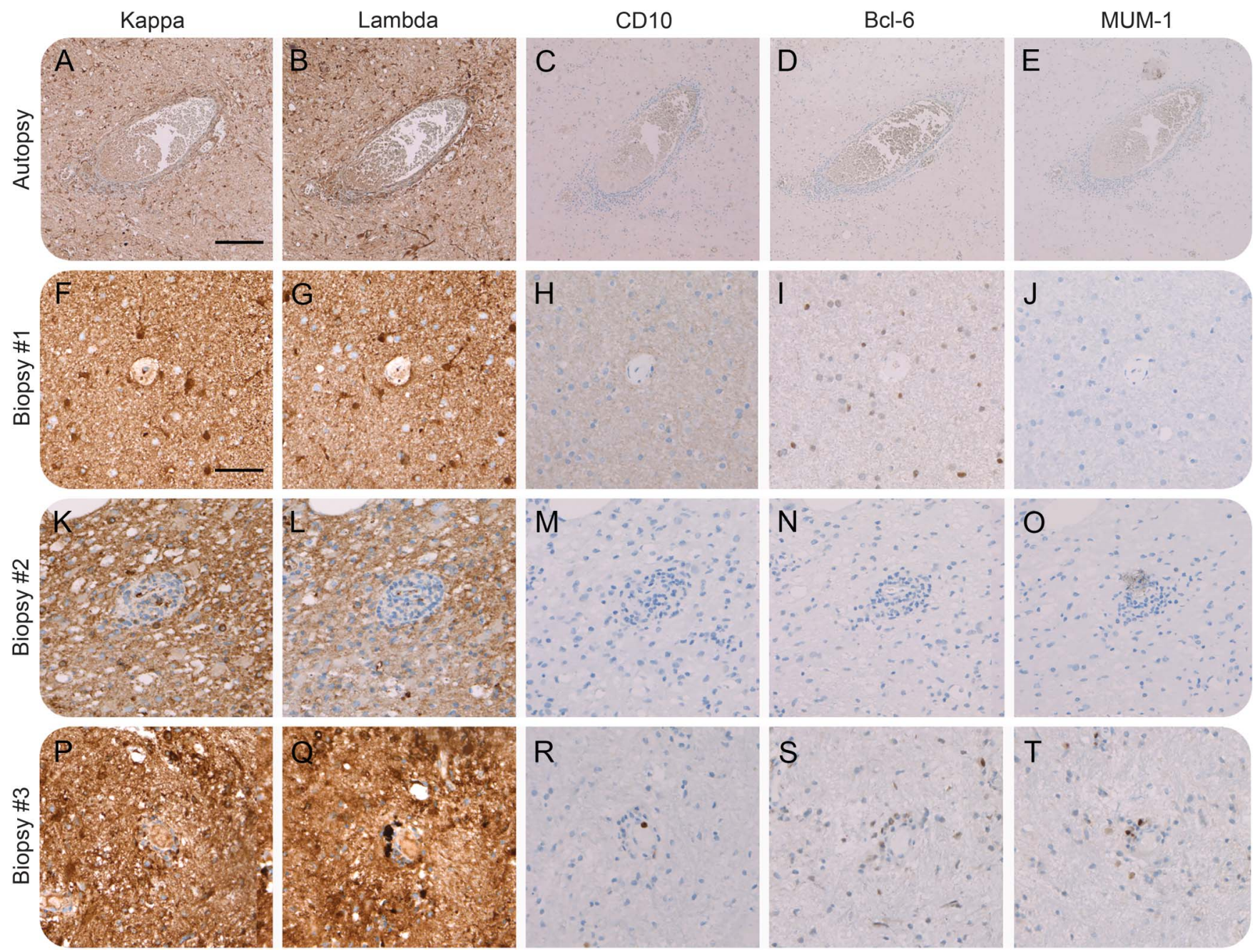

Immunohistochemical stainings for light chain restriction (lambda-smi and kappa-smi: A, B, F, G, K, L, P, and Q), CD10 (C, H, M, and R), Bcl-6 (D, I, N, and S), and MUM-1 $(E, J, O$, and $T)$ in samples from an autopsy $(A-E)$ and biopsy specimens of 3 patients $(F-J, K-O$, and $P-T)$. We found no evidence for light chain restriction or significant staining for lymphoma markers $\mathrm{CD} 10, \mathrm{Bcl}-6$, or MUM-1 suggesting a prelymphoma or lymphoma state. Magnification $5 \times(\mathrm{A}-\mathrm{E}), 10 \times$ (F-T). Scale bar: $200 \mu \mathrm{m}(\mathrm{A}-\mathrm{E}), 100 \mu \mathrm{m}(\mathrm{F}-\mathrm{T})$. 


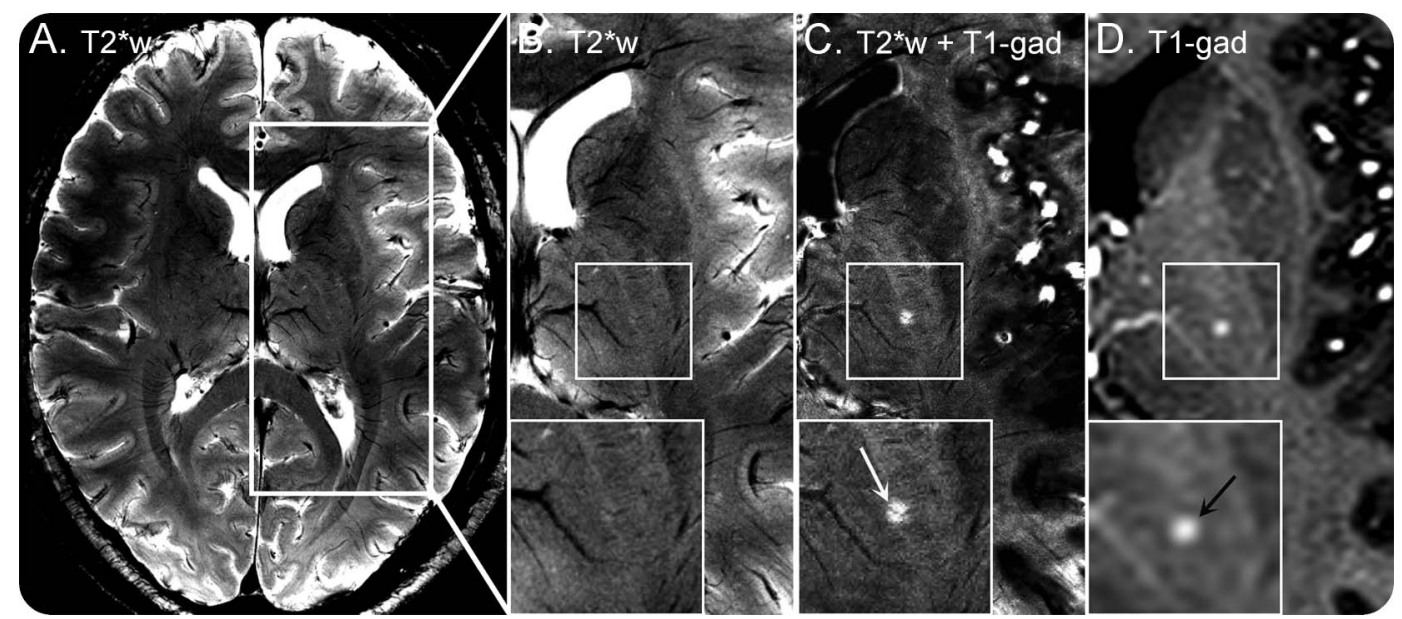

Supratentorial T2*-weighted (A, B) and postgadolinium T1-weighted images (D) of patient 5 obtained at 7.0T are displayed. Postgadolinium T1-weighted imaging depicts a small contrast-enhancing lesion (black arrow, D) that is only marginally delineated on corresponding T2*-weighted images (zoom, B) despite using a very high spatial resolution of $0.08 \mathrm{~mm}^{3}$. Fusion (C) of coregistered postgadolinium T1-weighted (D) and T2*-weighted (B) identifies a small brain vessel within the center of the chronic lymphocytic inflammation with pontine perivascular enhancement responsive to steroids (CLIPPERS) lesion (white arrow, C). T2* $\mathrm{w}=2 \mathrm{D} \mathrm{T} 2 *$-weighted fast low angle shot at 7.0T, echo time $(\mathrm{TE})=25.0 \mathrm{~ms}$, repetition time $(\mathrm{TR})=1,820$ $\mathrm{ms}$, spatial resolution $=0.2 \times 0.2 \times 2 \mathrm{~mm}^{3} ; \mathrm{T} 1$-gad $=$ postgadolinium 3D T1-weighted magnetization-prepared rapid gradient echo at $7.0 \mathrm{~T}, \mathrm{TE}=2.98 \mathrm{~ms}, \mathrm{TR}=2,300 \mathrm{~ms}$, inversion time $=900 \mathrm{~ms}$, spatial resolution $=1.0 \times 1.0 \times 1.0 \mathrm{~mm}^{3}$.

of $0.08 \mathrm{~mm}^{3}$ to display small $\mathrm{T} 2 * \mathrm{~W}$ hypointense venous structures within gadolinium-enhancing hyperintense CLIPPERS lesions (figure 3). Indeed, a distinct venous vessel could be seen within the center of the thalamic CLIPPERS lesion in vivo (figure 3C). Due to technical limitations, a different approach with a lower spatial resolution of $0.25 \mathrm{~mm}^{3}$ was used for infratentorial areas. Even with this approach, a very small venous vessel could be depicted within 8 of 10 contrast-enhancing CLIPPERS lesions on fused postgadolinium VIBE and SWI (data not shown).

In addition, 7.0T T1W MPRAGE depicted numerous T1W hypointense lesions within the pons and cerebellum (figure 4, E-G) in patient 5, which were not visible on conventional 3.0T MRI (figure 4, A-D), indicating severe tissue destruction within postinflammatory CLIPPERS lesions (figure 4). Multiple pontine postinflammatory T1W hypointense lesions were also found in patient 6 by using T1W imaging at $7.0 \mathrm{~T}$ (data not shown). Corresponding to the 7.0T MRI findings, neurofilament and myelin staining revealed axonal injury and myelin loss in brainstem areas with severe inflammation of the autopsy case (figure 4, $\mathrm{H}$ and I). Preservation of axons and myelin was seen in areas with limited inflammation (figure 4, $\mathrm{J}$ and $\mathrm{K}$ ).

DISCUSSION In this article, we describe neuropathologic as well as high-field/ultra-high-field MRI features of 6 patients with CLIPPERS syndrome.
The diagnosis of CLIPPERS syndrome was supported by (1) postcontrast T1-weighted MRI with typical punctate, nodular, or curvilinear and occasionally more confluent gadolinium-enhancing lesions, (2) perivascular inflammation in the brainstem dominated by $\mathrm{T}$ cells, (3) clinical course with several steroidresponsive relapses, and (4) absence of other diseases. In addition to punctate and curvilinear lesions, case 5 had a larger confluent lesion in the cerebellum. Similar lesions were previously described in patients with CLIPPERS ${ }^{2}$ and a biopsy of the cerebellar lesion of case 5 demonstrated histopathologic features of CLIPPERS.

We found widespread perivascular inflammation in brain areas with normal appearance on 3.0T MRI in an autopsy case of CLIPPERS syndrome. The inflammatory pattern of these regions was similar to the original ${ }^{1}$ and later neuropathologic descriptions of the disease $e^{2,3}$ characterized by pronounced CD4+ $\mathrm{T}$-cell infiltration of brain tissue and less predominant angiocentric CD8 + T-cell and CD20 + B-cell inflammation. Although T2W and gadolinium-enhancing lesions were found only in the pons, midbrain, and cerebellar regions, inflammation could be seen as distant as the parietal cortex and even involved the cranial nerve roots. The degree of inflammation, however, showed a gradient of less inflammation with greater distance from the primarily affected areas (brainstem $>$ cerebellum $>$ insular cortex $>$ parietal cortex), suggesting that the inflammation seen on conventional 1.5T-3.0T MRI only depicts the most severely 


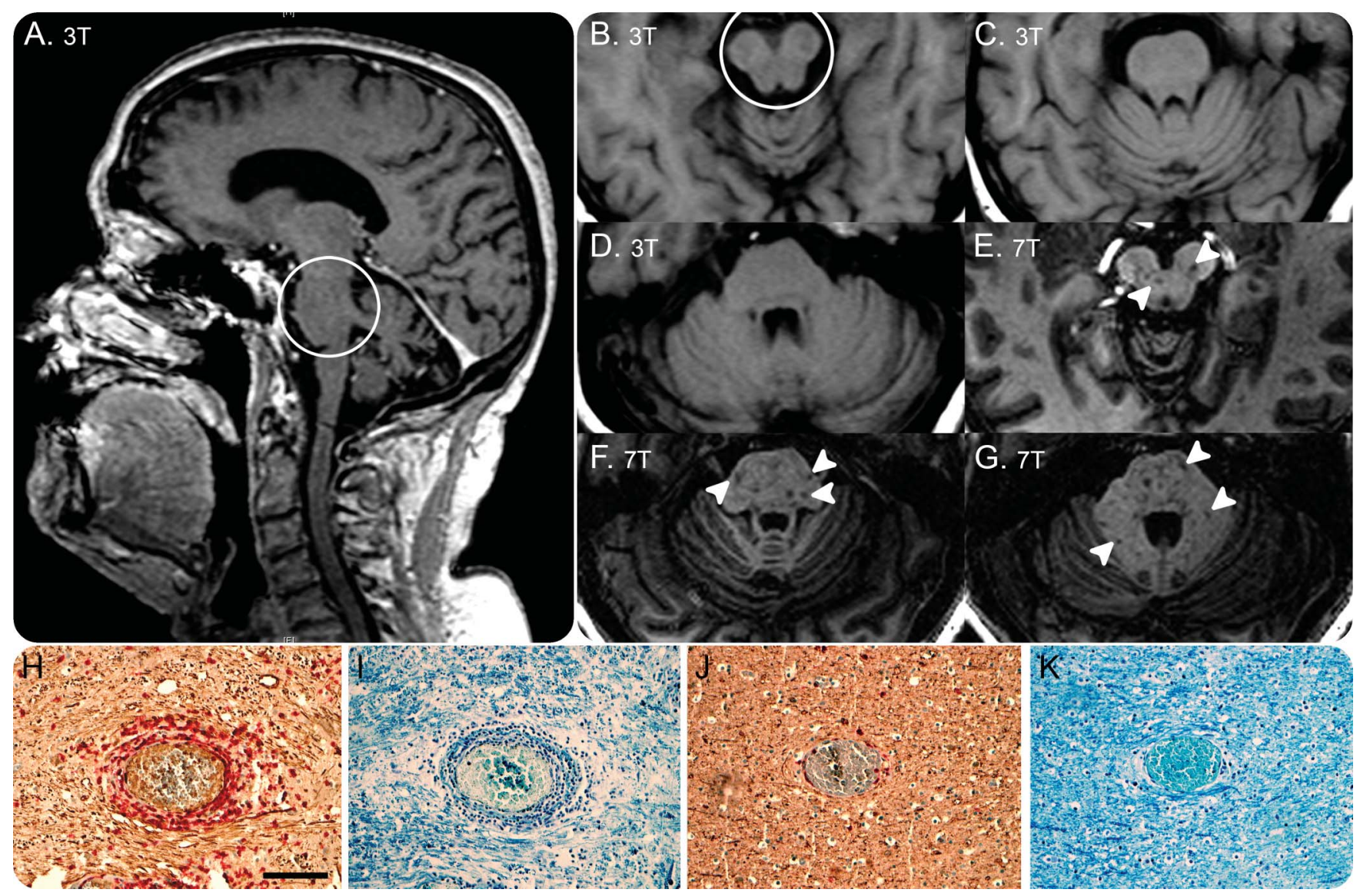

T1-weighted images of patient 5 at 3.0T and 7.0T are shown. Owing to substantially increased signal-to-noise ratio, 7.0T T1-weighted magnetization-prepared rapid gradient echo delineates numerous T1-weighted hypointense lesions within the pons and cerebellum (arrowheads, E-G). In contrast, corresponding areas appear normal (C, D) or only marginally hypointense on T1-weighted images at 3.0T (circle, A, B). T1-weighted hypointensity within non-contrast-enhancing chronic lymphocytic inflammation with pontine perivascular enhancement responsive to steroids lesions may indicate postinflammatory structural brain damage including axonal loss on top of demyelination. Immunohistochemical stainings of autopsy case for neurofilament (NF) revealed massive axonal injury in the inflammatory lesion of the brainstem ( $\mathrm{H} ; \mathrm{CD} 3$ red, NF brown) compared to insular cortex with limited inflammation (J). The presence of phosphorylated NF in the perinuclear cytoplasm of neurons suggests retrograde neuronal degeneration due to axonal injury in the lesions $(\mathrm{H})$. Similarly, severe loss of perivascular myelin was found in areas of severe inflammation (I), compared to areas with limited inflammation (K), with Luxol fast blue and hematoxylin \& eosin. 7.0T = 3D T1-weighted magnetization-prepared rapid gradient echo at $7.0 \mathrm{~T}$, echo time $=2.98 \mathrm{~ms}$, repetition time $=2,300 \mathrm{~ms}$, inversion time $=900 \mathrm{~ms}$, spatial resolution $=1.0 \times 1.0 \times 1.0 \mathrm{~mm}^{3}$; 3.0T $=$ standard T1-weighted axial images (B-D) and T1-weighted magnetization-prepared rapid gradient echo at 3.0T (A). 3.0T and 7.0T MRI examinations were performed 3 days apart. Magnification $10 \times(\mathrm{H}-\mathrm{K})$. Scale bar $=100 \mu \mathrm{m}$.

affected brain regions. Consistent with these neuropathologic findings, cognitive impairment such as dysexecutive syndromes, language disturbance, frontal release signs and cortical atrophy were described in a case series of CLIPPERS syndrome, suggesting that cortical involvement may be more prominent than initially thought. ${ }^{2}$

Since our pathologic examinations revealed widespread inflammatory changes in brain tissue appearing normal on conventional 3.0T MRI, we performed ultra-high-field 7.0T MRI in 2 additional CLIPPERS cases to investigate such possibilities in vivo. Indeed, these efforts revealed contrastenhancing lesions in supratentorial locations, supporting widespread inflammation in brain areas, in contrast to the only other autopsy report published recently. ${ }^{19}$ The number of such contrast-enhancing lesions was, however, limited, which is likely due to the immunosuppressive treatment: MRI changes in CLIPPERS respond rapidly to immunosuppression. ${ }^{13,20,21}$ Remarkably, when combining T1W and supratentorial $\mathrm{T} 2 * \mathrm{~W}$ images with a resolution as good as $0.08 \mathrm{~mm}^{3}$, we could show a vascular structure within most contrast-enhancing lesions. The 7.0T MRI thus enabled visualization of perivascular inflammation, the neuropathologic hallmark of CLIPPERS, in vivo. The hypointense appearance of these intralesional vascular structures on $\mathrm{T} 2 * \mathrm{~W}$ and SWI images indicates the presence of a paramagnetic material. Thus, these intralesional vascular structures are most likely small veins filled with paramagnetic deoxyhemoglobin. ${ }^{22}$ A central intralesional vein was previously reported to be a characteristic finding in MS lesions. ${ }^{11,12,14,16}$ On the contrary, a central vein is 
only rarely depictable in brain lesions of other origin such as neuromyelitis optica, ${ }^{14}$ cerebral small vessel disease, ${ }^{23}$ and Susac syndrome, ${ }^{16}$ which is considered a microangiopathy of the brain, cochlea, and retina. ${ }^{24-26}$ Evidence for the presence of MRIdetectable intralesional veins in other inflammatory vasculopathies such as Wegner granulomatosis, Behçet syndrome, primary CNS vasculitis, and vasculitis in systemic lupus erythematosus is, however, limited. Of note, highly resolving imaging techniques were needed to visualize the intralesional venous vessel in CLIPPERS lesions, which thus appear much smaller in diameter than central veins commonly visualized in MS lesions on $7.0 \mathrm{~T} \mathrm{~T} 2 * \mathrm{~W}$ images. However, as no systematic analysis of vessel diameters in CLIPPERS vs MS could be performed owing to the low sample size, future comparative studies will have to investigate whether this feature is consistently detectable in other CLIPPERS cases and distinct from MS.

In addition, we utilized the higher sensitivity of 7.0T T1W MPRAGE vs T1W imaging at $1.5 \mathrm{~T}^{15}$ and $3.0 \mathrm{~T}^{27}$ to detect brain lesions and to investigate brain areas that appeared normal at lower field strengths. Although 1.5T-3.0T MRI and 7.0T MRI were done at different time points, therefore direct comparison was not possible, patients had no signs of clinical activity at the time of the MRI examinations. Ultra-high-field MRI revealed numerous T1W hypointense lesions within the pons and cerebellum in one of the patients, indicating postinflammatory brain tissue destruction, which were not visible on conventional $3.0 \mathrm{~T}$ MRI. Indeed, axonal injury with evidence of axonal spheroids and torpedoes was found in our autopsy case, similar to previous results. ${ }^{2,3}$ However, axonal injury and myelin loss was observed only in areas with severe inflammation, which may explain the restricted localization of T1W hypointense lesions on 7.0T MRI.

Since emerging cases of CLIPPERS with subsequent diagnosis of lymphoma have been recently described, ${ }^{6-8}$ we investigated this possibility in the autopsy case and available biopsy samples from 3 other Danish cases. We found no evidence for prelymphoma or lymphoma when using immunohistochemical staining for light chain restriction or lymphoma markers CD10, Bcl-6, and MUM-1. Since the analyses were done on the initial diagnostic biopsies, this might not exclude per se that the patients progressed into a lymphoma. However, 3 of the patients have been already followed for a mean of more than 6 years without any evidence of lymphoma. Altogether, our present results strengthen the concept that CLIPPERS is a distinct disease entity that can be discerned from alternative diagnoses such as lymphoma or vasculitis.
We found perivascular inflammation predominated by $\mathrm{CD} 4+\mathrm{T}$ cells even in areas with normal appearance on 3.0T MRI and extending to the cranial nerve roots, suggesting widespread inflammation in CLIPPERS syndrome, though most prominent in the primarily affected brainstem. We found no evidence of lymphoma in our 4 investigated patients. When applying ultra-high-field MRI at 7.0T, supratentorial lesions could be confirmed, and most of the contrastenhancing lesions contained a vessel structure indicating the pathologic hallmark of CLIPPERS syndrome in vivo. The 7.0T MRI also indicated prominent $\mathrm{T} 1 \mathrm{~W}$ hypointensities suggesting tissue injury, which could not be seen on 3.0T MRI but corresponded to axonal injury and loss of myelin in the autopsy specimen. One limitation of the study might be the application of immunosuppressive treatment at the time of biopsy or MRI examination (table 1).

\section{AUTHOR CONTRIBUTIONS}

Morten Blaabjerg: design and conceptualization, clinical data, analysis and interpretation of the data, drafting and revising the manuscript. Klemens Ruprecht: MRI design and conceptualization, clinical data, analysis and interpretation of the data, drafting and revising the manuscript. Tim Sinnecker: MRI analysis and interpretation of the data, revising the manuscript. Daniel Kondziella: clinical data, interpretation of the data, revising the manuscript. Thoralf Niendorf: MRI analysis and interpretation of the data, revising the manuscript. Bjørg Morell Kerrn-Jespersen: clinical data, interpretation of the data, revising the manuscript. Mette Lindelof: clinical data, interpretation of the data, revising the manuscript. Hans Lassmann: pathological analysis and interpretation of the data, revising the manuscript. Bjarne Winther Kristensen: pathological analysis and interpretation of the data, revising the manuscript. Friedemann Paul: MRI design and conceptualization, analysis and interpretation of the data, drafting and revising the manuscript. Zsolt Illes: design and conceptualization, analysis and interpretation of the data, drafting and revising the manuscript.

\section{STUDY FUNDING}

Lundbeckfonden and Scleroseforeningen (Denmark) to Z.I.; Deutsche Forschungsgemeinschaft (Exc 257) and the German Ministry for Education and Research (BMBF Competence Network Multiple Sclerosis) to F.P.; German Ministry for Education and Research (BMBF Competence Network Multiple Sclerosis) to K.R.

\section{DISCLOSURE}

M. Blaabjerg reports no disclosures. K. Ruprecht served on the scientific advisory board for Sanofi-Aventis/Genzyme, Novartis, and Roche; received travel funding and/or speaker honoraria from Bayer Healthcare, Biogen Idec, Merck Serono, Sanofi-Aventis/Genzyme, Teva Pharmaceuticals, Novartis, and Guthy Jackson Charitable Foundation; is an academic editor for PLOS ONE; receives publishing royalties from Elsevier; and received research support from Novartis and the German Ministry of Education and Research. T. Sinnecker received travel funding from Bayer, Teva, Novartis, and Genzyme. D. Kondziella received speaker honoraria and/or travel funding from Bristol Meyer Squibb and UCB and has consulted for Pfizer Sweden. T. Niendorf received travel funding and/or speaker funding from Siemens Healthcare, is founder and CEO of MRI TOOLS GmbH, and received research support from Siemens Healthcare and Helmholtz Alliance. B.M. Kerrn-Jespersen received travel funding from Biogen. M. Lindelof reports no disclosures. H. Lassmann received travel funding and speaker honoraria from Biogen Idec, Novartis, and Teva; is on the editorial board for several journals in the fields of neurology and neuroscience; has consulted for Biogen Idec and Amgen; and received research support from Austrian Science Fund, European 
Union. B.W. Kristensen reports no disclosures. F. Paul is on the steering committees for Novartis and MedImmune; received speaker honoraria and travel funding from Bayer, Novartis, Biogen Idec, Teva, Sanofi-Aventis/Genzyme, and Merck Serono; is an academic editor for PLoS ONE; is an associate editor for Neurology: Neuroimmunology \& Neuroinflammation; has consulted for SanofiGenzyme, BiogenIdec, and MedImmune; and received research support from Bayer, Novartis, Biogen Idec, Teva, Sanofi-Aventis/Genzyme, Merck Serono, German Research Council, Werth Stiftung of the City of Cologne, German Ministry of Education and Research, Arthur Arnstein Stiftung Berlin, EU FP7 Framework Program, Guthy Jackson Charitable Foundation, and National Multiple Sclerosis Society of the USA. Z. Illes served on the scientific advisory board for Biogen Idec, Novartis, Sanofi-Aventis/ Genzyme, and Teva Pharmaceuticals; received travel funding and/ or speaker honoraria from Biogen Idec, Novartis, Sanofi-Aventis/ Genzyme, Teva Pharmaceuticals, Bayer Healthcare, and Merck Serono; is on the editorial board for Clinical and Experimental Neuroimmunology; holds a patent for Fumaric acide derivates for medical use; and received research support from Biogen Idec, Region of Southern Denmark, Odense University Hospital, Scleroseforeningen, Lundbeckfonden, Direktor Enjar Jonasson, kaldet Jonsen, og Hustru's Mindelegat. Go to Neurology.org/nn for full disclosure forms.

Received November 10, 2015. Accepted in final form February 12, 2016.

\section{REFERENCES}

1. Pittock SJ, Debruyne J, Krecke KN, et al. Chronic lymphocytic inflammation with pontine perivascular enhancement responsive to steroids (CLIPPERS). Brain 2010;133: 2626-2634.

2. Simon NG, Parratt JD, Barnett MH, et al. Expanding the clinical, radiological and neuropathological phenotype of chronic lymphocytic inflammation with pontine perivascular enhancement responsive to steroids (CLIPPERS). J Neurol Neurosurg Psychiatry 2012;83:15-22.

3. Taieb G, Duflos C, Renard D, et al. Long-term outcomes of CLIPPERS (chronic lymphocytic inflammation with pontine perivascular enhancement responsive to steroids) in a consecutive series of 12 patients. Arch Neurol 2012; 69:847-855.

4. Buttmann M, Metz I, Brecht I, Brück W, WarmuthMetz M. Atypical chronic lymphocytic inflammation with pontocerebellar perivascular enhancement responsive to steroids (CLIPPERS), primary angiitis of the CNS mimicking CLIPPERS or overlap syndrome? A case report. J Neurol Sci 2013;324:183-186.

5. Ferreira RM, Machado G, Souza AS, Lin K, CorrêaNeto Y. CLIPPERS-like MRI findings in a patient with multiple sclerosis. J Neurol Sci 2013;327:61-62.

6. De Graaff HJ, Wattjes MP, Rozemuller-Kwakkel AJ, Petzold A, Killestein J. Fatal B-cell lymphoma following chronic lymphocytic inflammation with pontine perivascular enhancement responsive to steroids. JAMA Neurol 2013;70:915-918.

7. Lin AW, Das S, Fraser JA, et al. Emergence of primary CNS lymphoma in a patient with findings of CLIPPERS Can J Neurol Sci 2014;41:528-529.

8. Taieb G, Uro-Coste E, Clanet M, et al. A central nervous system B-cell lymphoma arising two years after initial diagnosis of CLIPPERS. J Neurol Sci 2014;344:224-226.

9. Müller K, Kuchling J, Dörr J, Harms L, Ruprecht K, Niendorf T. Detailing intra-lesional venous lumen shrinking in multiple sclerosis investigated by sFLAIR MRI at 7 T. J Neurol 2014;261:2032-2036.

10. Sinnecker T, Bozin I, Dörr J, et al. Periventricular venous density in multiple sclerosis is inversely associated with T2 lesion count: a 7 Tesla MRI study. Mult Scler 2013;19: 316-325.

11. Tallantyre EC, Brookes MJ, Dixon JE, Morgan PS, Evangelou N, Morris PG. Demonstrating the perivascular distribution of MS lesions in vivo with 7-Tesla MRI. Neurology 2008;70:2076-2078.

12. Kollia K, Maderwald S, Putzki N, et al. First clinical study on ultra-high-field MR imaging in patients with multiple sclerosis: comparison of $1.5 \mathrm{~T}$ and 7T. AJNR Am J Neuroradiol 2009;30:699-702.

13. Gaitán MI, de Alwis MP, Sati P, Nair G, Reich DS. Multiple sclerosis shrinks intralesional, and enlarges extralesional, brain parenchymal veins. Neurology 2013;80: 145-151.

14. Sinnecker T, Dörr J, Pfueller CF, et al. Distinct lesion morphology at 7-T MRI differentiates neuromyelitis optica from multiple sclerosis. Neurology 2012;79: 08-714.

15. Sinnecker T, Mittelstaedt P, Dörr J, et al. Multiple sclerosis lesions and irreversible brain tissue damage: a comparative ultrahigh-field strength magnetic resonance imaging study. Arch Neurol 2012;69:739-745.

16. Wuerfel J, Sinnecker T, Ringelstein EB, et al. Lesion morphology at 7 Tesla MRI differentiates Susac syndrome from multiple sclerosis. Mult Scler 2012;18:1592-1599.

17. Kerrn-Jespersen BM, Lindelof M, Illes Z, et al. CLIPPERS among patients diagnosed with non-specific CNS neuroinflammatory diseases. J Neurol Sci 2014;343:224-227.

18. List J, Lesemann A, Wiener E, et al. A new case of chronic lymphocytic inflammation with pontine perivascular enhancement responsive to steroids. Brain 2011;134:e185.

19. Moreira I, Cruto C, Correia C, Alves JE, Taipa R, Pires MM. Chronic lymphocytic inflammation with pontine perivascular enhancement responsive to steroids (CLIPPERS): postmortem findings. J Neuropathol Exp Neurol 2015;74:186-190.

20. Kastrup O, van de Nes J, Gasser T, Keyvani K. Three cases of CLIPPERS: a serial clinical, laboratory and MRI follow-up study. J Neurol 2011;258:2140-2146.

21. Sempere AP, Mola S, Martin-Medina P, Bernabeu A, Khabbaz E, Lopez-Celada S. Response to immunotherapy in CLIPPERS: clinical, MRI, and MRS follow-up. J Neuroimaging 2013;23:254-255.

22. Ge Y, Zohrabian VM, Osa EO, et al. Diminished visibility of cerebral venous vasculature in multiple sclerosis by susceptibility-weighted imaging at 3.0 Tesla. J Magn Reson Imaging 2009;29:1190-1194.

23. Tallantyre EC, Dixon JE, Donaldson I, et al. Ultra-highfield imaging distinguishes MS lesions from asymptomatic white matter lesions. Neurology 2011;76:534-539.

24. Sinnecker T, Kuchling J, Dusek P, et al. Ultrahigh field MRI in clinical neuroimmunology: a potential contribution to improved diagnostics and personalised disease management. EPMA J 2015;6:16. eCollection.

25. Dörr J, Krautwald S, Wildemann B, et al. Characteristics of Susac syndrome: a review of all reported cases. Nat Rev Neurol 2013;9:307-316.

26. Dörr J, Radbruch H, Bock M, et al. Encephalopathy, visual disturbance and hearing loss-recognizing the symptoms of Susac syndrome. Nat Rev Neurol 2009;5: 683-688.

27. Mistry N, Tallantyre EC, Dixon JE, et al. Focal multiple sclerosis lesions abound in "normal appearing white matter." Mult Scler 2011;17:1313-1323. 


\title{
Neurology \\ Neuroimmunology \& Neuroinflammation
}

\author{
Widespread inflammation in CLIPPERS syndrome indicated by autopsy and \\ ultra-high-field 7T MRI \\ Morten Blaabjerg, Klemens Ruprecht, Tim Sinnecker, et al. \\ Neurol Neuroimmunol Neuroinflamm 2016;3; \\ DOI 10.1212/NXI.0000000000000226
}

This information is current as of April 20, 2016

\section{Updated Information \& \\ Services}

Supplementary Material

References

Subspecialty Collections

Permissions \& Licensing

Reprints including high resolution figures, can be found at:

http://nn.neurology.org/content/3/3/e226.full.html

Supplementary material can be found at:

http://nn.neurology.org/content/suppl/2016/04/20/3.3.e226.DC1

This article cites 27 articles, 2 of which you can access for free at: http://nn.neurology.org/content/3/3/e226.full.html\#\#ref-list-1

This article, along with others on similar topics, appears in the following collection(s):

Autoimmune diseases

http://nn.neurology.org//cgi/collection/autoimmune_diseases

MRI

http://nn.neurology.org//cgi/collection/mri

Information about reproducing this article in parts (figures,tables) or in its entirety can be found online at:

http://nn.neurology.org/misc/about.xhtml\#permissions

Information about ordering reprints can be found online:

http://nn.neurology.org/misc/addir.xhtml\#reprintsus

Neurol Neuroimmunol Neuroinflamm is an official journal of the American Academy of Neurology.

Published since April 2014, it is an open-access, online-only, continuous publication journal. Copyright $(\subseteq$ 2016 American Academy of Neurology. All rights reserved. Online ISSN: 2332-7812.

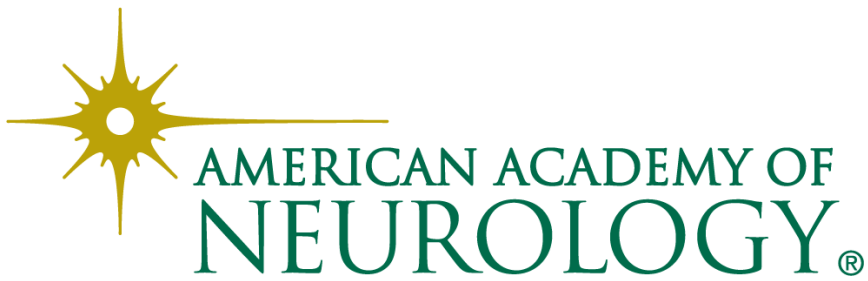

